\title{
VECTOR KIRCHHOFF ANALYSIS FOR THE STUDY OF RECTANGULAR MICROSTRIP ANTENNAS IMMERSED IN A PLASMA
}

\author{
PREM BHUSHAN MITAL, FIETE \\ Centre for Applied Research in Electronics, Indian Institute of Technology, New Delhi-110 016, \\ INDIA
}

(Received April 23, 1994; in final form May 11, 1994)

The radiation properties of a microstrip antenna immersed in an ionized medium have since been studied using potential function technique and linearized hydrodynamic theory. ${ }^{3}$ In the present paper, Vector Kirchhoff Analysis has been used for the study of rectangular microstrip antenna immersed in a plasma media. Expressions for radiated far fields have been obtained using this analysis. The analysis is mathematically precise and is used by reasonably approximating the actual aperture field distributions. The effect of ion sheath in the vicinity of a rectangular microstrip antenna is also discussed.

\section{INTRODUCTION}

It has been established that when an antenna is immersed in a plasma medium, an electroacoustic wave is generated in addition to the usual electromagnetic wave. The fields are separated into em and plasma modes. The em mode is the usual electromagnetic mode with an electric and magnetic field and gives a transverse em wave in the far zone of the antenna, whereas the plasma mode has an electric field but no magnetic field. The electroacoustic wave becomes a longitudinal plasma wave in the far zone of the antenna.

The radiation properties of the antenna are modified in a plasma medium to a great extent. ${ }^{1.4}$ The total radiation resistance of an antenna in a plasma consists of the sum of the radiation resistance due to electromagnetic $(\mathrm{em})$ mode $\mathrm{Re}$ and the radiation resistance due to the plasma $(\mathrm{P})$ mode $\mathrm{Rp}$.

\section{FORMULATION OF THE PROBLEM}

A general solution of the field equations in terms of the sources distributed through a region $\mathrm{v}$ and from fields on the surfaces for a time-periodic field:

$$
\begin{aligned}
\overline{\mathrm{E}}_{\mathrm{p}}=- & \frac{1}{4 \pi} \int_{\mathrm{v}}\left(\mathrm{j} \omega \mu \psi \overline{\mathrm{J}}+\overline{\mathrm{J}} \mathrm{m} \times \nabla \psi-\frac{\rho}{\epsilon} \nabla \psi\right) \mathrm{dv} \\
& +\frac{1}{4 \pi} \int_{\mathrm{S}_{1}+\mathrm{S}_{2} \ldots+\mathrm{S}_{\mathrm{n}}}[-\mathrm{j} \omega \mu \psi(\overline{\mathrm{n}} \times \overline{\mathrm{H}})+(\overline{\mathrm{n}} \times \overline{\mathrm{E}}) \times \nabla \psi+(\overline{\mathrm{n}} \cdot \overline{\mathrm{E}}) \nabla \psi] \overline{\mathrm{ds}}
\end{aligned}
$$


With Er, Hr denoting the components of scattered fields over the volume considered and transforming the integrals, the expression can be reduced to:

$E_{s}(P)=\frac{1}{4 \pi j \omega \epsilon} \int_{A}\left[k^{2}(\overline{\mathrm{n}} \times \overline{\mathrm{Hr}}) \psi+(\overline{\mathrm{n}} \times \overline{\mathrm{Hr}}) \cdot \nabla(\nabla \psi)+\mathrm{j} \omega \epsilon(\overline{\mathrm{n}} \times \overline{\mathrm{E}} \mathrm{r}) \times \nabla \psi\right] \mathrm{ds}$

Finally, carrying through the reduction of the integrals for the Fraunhofer region (far-zone field) and inserting electric and magnetic currents in terms of fields, we arrive at

$E_{s}(P)=-\frac{j k}{4 \pi R} e^{-j k R} \bar{R}_{1} \times \int_{A}\left[\bar{n} \times \bar{E}_{r}-\sqrt{\frac{\mu}{\epsilon}} \bar{R}_{1} \times(\bar{n} \times \overline{\mathrm{H}} r)\right] \cdot \mathrm{e}^{j k \bar{\rho} \cdot R l} \mathrm{ds}$.

where:

$\mathrm{k}=\frac{2 \pi}{\lambda_{0}}=\omega\left(\mu_{0} \epsilon_{0}\right)^{1 / 2}$

$\mathrm{R}_{1}=$ Unit vector from origin to the field point in the direction $\theta, \phi$

$\hat{\mathbf{n}}=$ Outward unit vector normal to a surface.

$\bar{\rho}=$ Vector from origin of the coordinate system to the element ds of the aperture area.

$\overline{\mathrm{E}} \mathrm{r}=$ Electric field over the aperture

$\overline{\mathrm{H}} \mathrm{r}=$ Magnetic field over the aperture

$\mathrm{A}=$ Aperture Area.

Resultant far-zone fields are:

Electromagnetic mode:

$$
\begin{aligned}
& E_{\theta}=\frac{-2 j k E y}{4 \pi r} e^{-j \beta 0 A r} \cdot \operatorname{Fn}\left(A \beta_{0}\right)\left[\left\{k\left(\epsilon_{\text {eff }}\right)^{1 / 2} \cos \theta \cos \xi\right.\right. \\
& \left.+\mathrm{jA} \sin \xi\}-\Gamma\left\{\mathrm{k}\left(\epsilon_{\mathrm{eff}}\right)^{1 / 2} \cos \theta \cos \xi-\mathrm{jA} \sin \xi\right\}\right] \sin \phi . \\
& E_{\phi}=\frac{-2 j k E y}{4 \pi r} e^{-j \beta) A r} \cdot \operatorname{Fn}\left(A \beta_{0}\right)\left[\left\{k\left(\epsilon_{\text {eff }}\right)^{1 / 2} \cos \xi\right.\right. \\
& \left.+\mathrm{jA} \cos \theta \sin \xi\}-\Gamma\left\{\mathrm{k}\left(\epsilon_{\mathrm{eff}}\right)^{1 / 2} \cos \xi-\mathrm{jA} \cos \theta \sin \xi\right\}\right] \cos \phi
\end{aligned}
$$

\section{Electroacoustics Mode:}

$$
\mathrm{E}_{\mathrm{p}}=\frac{2 \mathrm{jEy}\left(1-\mathrm{A}^{2}\right)}{\epsilon_{0} \pi \omega \mathrm{r} \mathrm{A}^{2}} \beta_{\mathrm{p}} \mathrm{e}^{-\mathrm{j} \beta \mathrm{pr}} \mathrm{Fn}(\beta \mathrm{p})[\cos (\beta \mathrm{p} \cos \theta-\beta) 1]
$$




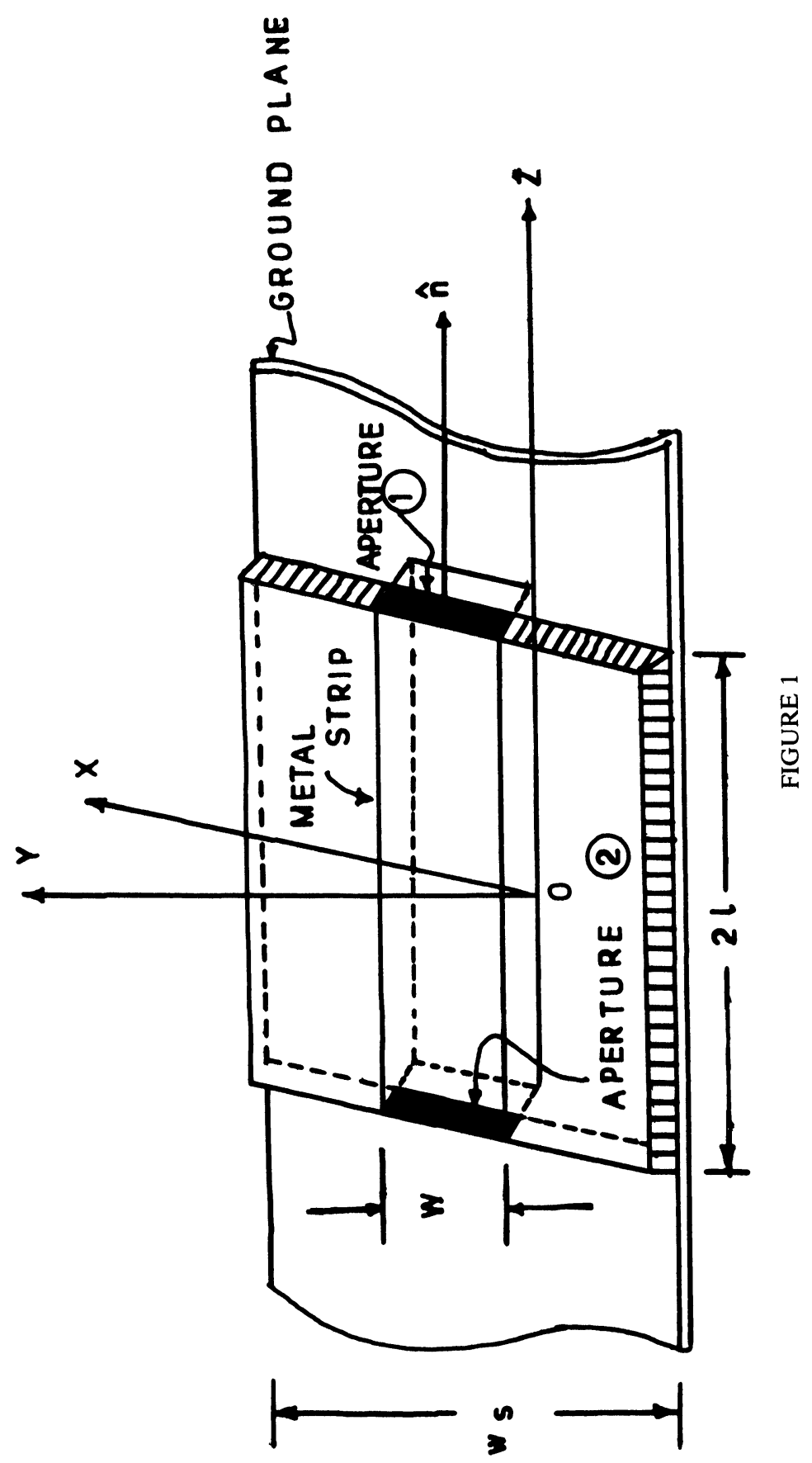


where:

$\mathrm{Fn}(\mathrm{x})=-2 \mathrm{hw} \operatorname{sinc}\left[\mathrm{x} \frac{1}{2} \omega \sin \theta \cos \phi\right] \operatorname{sinc}[\mathrm{xh} \sin \theta \sin \phi] \psi$

$\psi=\mathrm{e}^{\mathrm{j} \beta \mathrm{e}} \sin \theta(1 / 2 \omega \cos \phi+\mathrm{h} \sin \phi)$

$\xi=\mathrm{kl}\left[\mathrm{A} \cos \theta-\left(\epsilon_{\mathrm{eff}}\right)^{1 / 2}\right]$

\section{ION SHEATH EFFECTS IN THE VICINITY OF RECTANGULAR} ANTENNA

When a microstrip antenna is immersed in an ionized medium, electrons are displaced and move away from the patch conductor. This motion of electrons produces a polarization field due to election deficiency. An equilibrium condition is attained forming a positive ion sheath adjacent to the patch conductor. The sheath shields the medium from the effects of any charge on the antenna. Assuming an equivalent sharp boundary or sheath edge, the capacity of the antenna to this surface is $\mathrm{C}=$ $\epsilon_{0} \mathrm{~A} / \mathrm{d}$ farads.

where $\mathrm{A}=$ Area of patch conductor

$\epsilon_{0}=1 / 36 \pi 10^{9}$ farads per meter

Thus, the charge $\mathrm{Q}$ on the antenna is $\mathrm{Q}=\epsilon_{0} \mathrm{~A} \mathrm{~V} / \mathrm{d}$ or $\rho_{\mathrm{s}}=\mathrm{Q} / \mathrm{A}=\epsilon_{0} \mathrm{~V} / \mathrm{d}$. For a thin rectangular patch carrying a uniform charge $\rho \mathrm{S} / \mathrm{m}^{2}$ assuming negligible magnetic field, the $\mathrm{z}$-directed electric field is given as

$\overline{\mathrm{E}}=\frac{\rho_{s}}{\pi \epsilon_{0}} \tan ^{-1}\left[\frac{\mathrm{ab}}{\mathrm{d}\left(\mathrm{a}^{2}+\mathrm{b}^{2}+\mathrm{d}^{2}\right)^{1 / 2}}\right] \begin{aligned} & -\mathrm{a} \leq \mathrm{x} \leq \mathrm{a} \\ & \hat{\mathrm{z}}_{\mathrm{z}}-\mathrm{b} \leq \mathrm{y} \leq \mathrm{b}\end{aligned}$

Equating this field to the polarization field arising due to electron deficiency, values of sheath size for different values of $\mathrm{V} / \mathrm{N}$ are obtained.

$\frac{\mathrm{V}}{\mathrm{d}}=\frac{\epsilon \mathrm{Nd}}{\pi \epsilon_{0}} \tan ^{-1}\left[\frac{\mathrm{ab}}{\mathrm{d}\left(\mathrm{a}^{2}+\mathrm{b}^{2}+\mathrm{d}^{2}\right)^{1 / 2}}\right]$

Where $\mathrm{V}=$ Antenna Potential in volts

$\mathrm{N}=$ Number of electrons percubic meter.

From, this, the thickness $d$ of the sheath is obtained for different values of $\mathrm{V} /$ N.

\section{ACKNOWLEDGMENT}

The author is indebted to Prof. B. Bhat and Prof. S.K. Koul, Centre for Applied Research in Electronics for providing research facilities. 


\section{REFERENCES}

1. K.M. Chen, Interaction of a radiating source with a plasma. Proc. IEE. Oct. 1964 pp. 1668-1678.

2. A.G. Derneryd, A theoretical investigation of the rectangular microstrip Antenna IEEE Trans. vol. AP 26, July 78, pp. 532-535.

3. R.K. Gupta, Plasma and its effect on Radiations from different types of Antennas I.E.T.E. Journal vol. 8, No. 3, 1967, pp. 181-206.

4. J.R. Wait, Radiation of Electromagnetic and electroacoustics waves in a plasma. part I. Applied Sc. Res. 1965, pp. 413-432. 

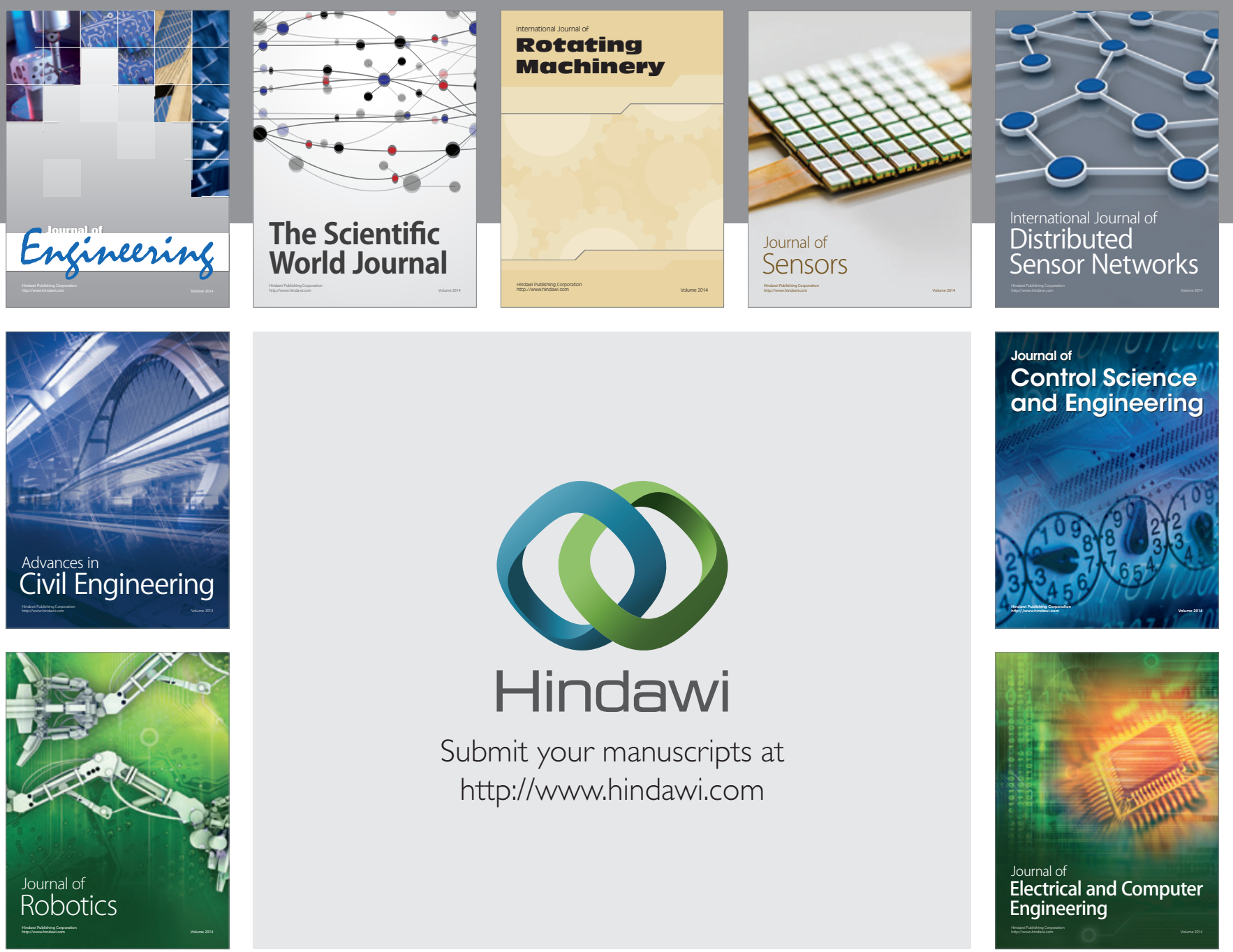

Submit your manuscripts at

http://www.hindawi.com
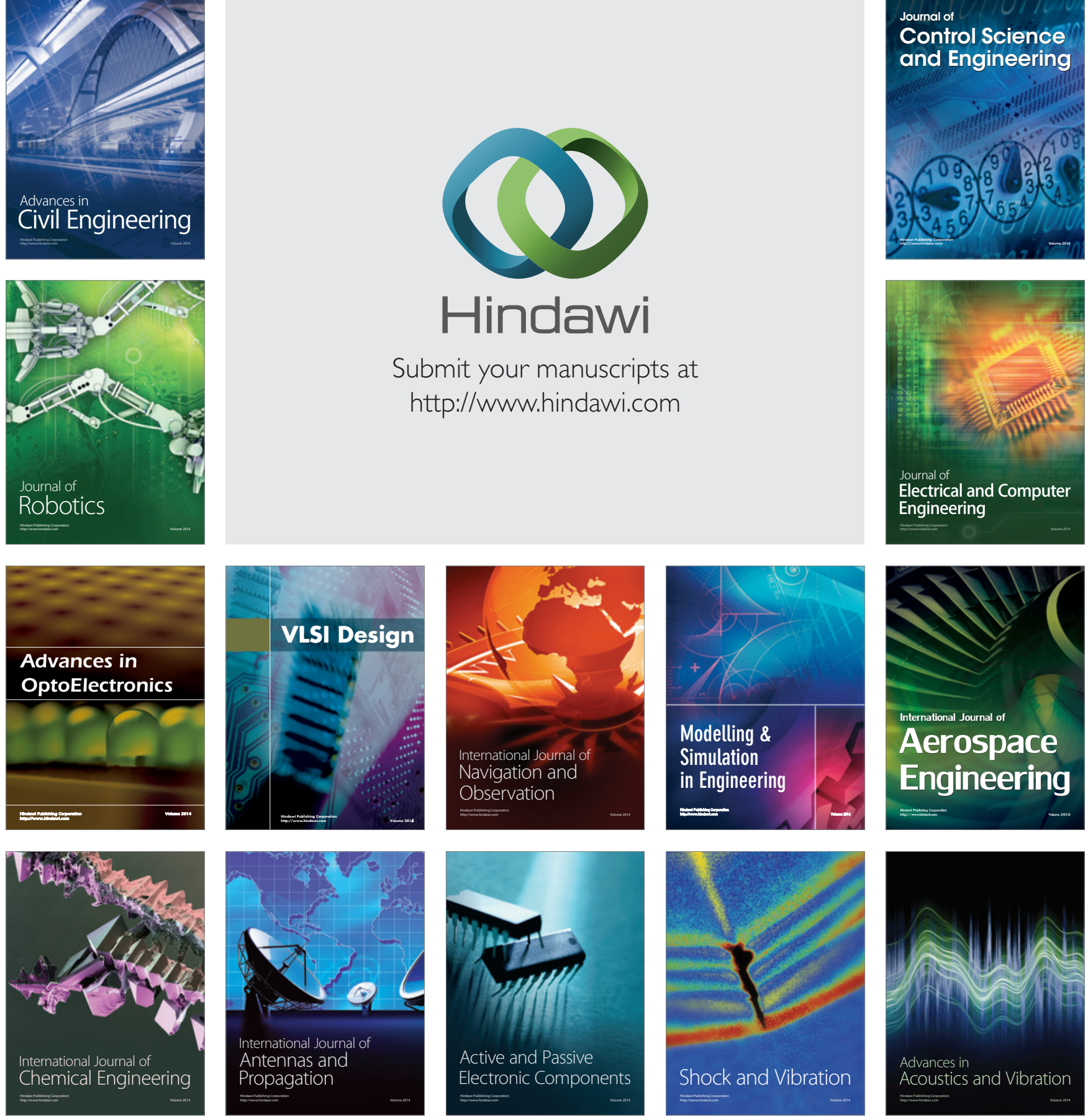